RUNHETC 2001-05

\title{
Form factors of soliton-creating operators in the sine-Gordon model
}

\author{
Sergei Lukyanov and Alexander Zamolodchikov \\ Department of Physics and Astronomy, Rutgers University \\ Piscataway, NJ 08855-0849, USA \\ and \\ L.D. Landau Institute for Theoretical Physics, \\ Chernogolovka, 142432, RUSSIA
}

\begin{abstract}
We propose explicit expressions for the form factors, including their normalization constants, of topologically charged (or soliton-creating) operators in the sine-Gordon model. The normalization constants, which constitute the main content of our proposal, allow one to find exact relations between the short- and long-distance asymptotics of the correlation functions. We make predictions concerning asymptotics of fermion correlation functions in the massive Thirring model, $S U(2)$-Thirring model with anisotropy, and in the half-filled Hubbard chain.
\end{abstract}

February, 01 


\section{Introduction}

Exact form factors are of significant interest in integrable quantum field theories in two space-time dimensions, and much progress has been made during the last 25 years in computing these quantities in various models, and in their applications to the analysis of correlation functions. By $n$-particle form factors we understand as usual the matrix elements of any local (or "semi-local", see below) field operator $\mathcal{O}(x)$ between the vacuum and the states containing $n$ particles. One of the reasons for the interest lies in the fact that exact form factors allow one to generate large-distance expansions for the correlation functions by inserting a complete set of states of asymptotic particles. If the factorizable $S$-matrix of these particles is known, the form factors are determined by solving the "form factor bootstrap equations" (see [1] for coherent exposition of the bootstrap program. Refs.[2] includes some early works on the subject).

It is important to note that these form factor bootstrap equations constitute a linear system, i.e. if a certain collection of form factors solves the equations, the renormalized collection (with all form factors associated with the same operator $\mathcal{O}$ multiplied by the same constant) also does; this of course represents the freedom in normalization of the field operator $\mathcal{O}$. On the other hand it is usually convenient to fix the normalizations of the field operators in terms of the short-distance behavior of their correlation functions. If the short-distance behaviour is controlled by associated CFT, the two-point correlation function of a spinless field $\mathcal{O}(x)$ has the asymptotic form

$$
\left\langle\mathcal{O}(x) \mathcal{O}^{\dagger}(0)\right\rangle \rightarrow \frac{1}{|x|^{4 \Delta_{\mathcal{O}}}} .
$$

Conventional choice of the coefficient 1 in the power law (1.1) fixes (up to a phase, which usually can be fixed through other correlation functions) the normalization of $\mathcal{O}$. The problem arises of finding the specific normalization of the form factors which corresponds to the "CFT normalization" (1.1).

In principle, this problem can be solved by analyzing the short-distance behavior of the form factor expansion. However, in practice it is usually possible to compute only the first few terms of this expansion. While yielding in many cases excellent numerical data even for rather small distances (see e.g. [3]), this truncated series does not provide exact analytic information about the coefficient in the short-distance asymptotic (1.1). Therefore the problem of determining the "CFT normalizations" of the form factors for a generic operator $\mathcal{O}$ remains largely open (notable exceptions being the cases when $\mathcal{O}$ is a component of some 
conserved current, e.g. energy-momentum tensor, and the normalizations of its form factors can be fixed through the Ward identities, see e.g. [1]). Some progress was made in [4] where the expectation values of topologically neutral primary operators in the sine-Gordon model (and in some other integrable QFT [5,66,7] ) were determined. This result makes it straightforward to fix the normalizations of all higher form factors of these operators using Smirnov's "annihilation pole" relation [1] of the form factor bootstrap [8].

In this paper we extend the above result to a class of topologically charged fields in the sine-Gordon model. Obviously, the vacuum expectation values of fields with nonzero topological charge vanish. The simplest form factor of an operator carrying topological charge $n$ is its matrix element between the vacuum and an $n$-soliton (or $(-n)$-antisoliton, if $n$ is negative) state. In Section 3 we propose an explicit expression for these simplest form factors of "CFT-normalized" primary operators. Our proposal, Eq.(3.1) below, actually concerns the corresponding normalization factor $\mathbf{Z}_{n}$ in (2.12) (without regard of normalization, some form factors with $n \neq 0$ were considered before in Refs. [9]). In Section 4 we carry out various checks of this conjectured expression.

Let us mention here that there is a one-parameter family of primary fields of given topological charge $n$ (see Sect. 2 for details), and for generic values of the parameter $a$ these fields (denoted $\mathcal{O}_{a}^{n}$ henceforth) are not local (they are "semi-local" in the terminology of [10]). Nonetheless, there are several important reasons to be interested in form factors and correlation functions of such nonlocal operators. Let us mention a few. First, specific discrete values of the parameter $a$ provide a set of local topologically charged fields which in fact coincide with the fundamental Fermi fields and their composites in the massive Thirring model. For other discrete values of this parameter these fields become components of conserved nonlocal currents generating the affine quantum group symmetry of the sineGordon model. These relations will be used in Sect.4. Second, analytic properties in the parameter $a$ are likely to be illuminating, as was demonstrated in [5] for expectation values of neutral fields. We will say some words on this subject in Sect.6. Finally, in many cases it has proved to be useful to factorize local fields into nonlocal ones. For example, the $S U(2)$ Thirring model (and its anisotropic deformation) can be bosonized in terms of a sine-Gordon field plus a free massless boson, the correlation functions of basic Fermi fields in this model being expressed through the correlators of the nonlocal fields of the sineGordon theory. In Sect.5 we use this relation to make predictions about exact asymptotics of fermion correlation functions in the $S U(2)$ Thirring and Hubbard models. 


\section{Topologically charged fields in the sine-Gordon model}

The sine-Gordon model, which is described by the euclidean action 1

$$
\mathcal{A}_{s G}=\int d^{2} x\left\{\frac{1}{16 \pi}\left(\partial_{\nu} \varphi\right)^{2}-2 \mu \cos (\beta \varphi)\right\}
$$

is invariant w.r.t. the field translations $\varphi(x) \rightarrow \varphi(x) \pm 2 \pi / \beta$. As a well known consequence, the model admits a class of fields creating nonzero topological (or soliton) charge. The simplest of these fields can be described as the "dual exponentials"

$$
\mathcal{O}_{0}^{n}(x)=e^{\frac{n}{4 \beta} \int_{\mathcal{C}_{x}} \epsilon_{\mu \nu} \partial^{\mu} \varphi d x^{\nu}}
$$

with integer $n$. Here the integration goes along some contour $\mathcal{C}_{x}$ ("Dirac string") starting at infinity and ending at $x$, the precise shape of this contour being very much arbitrary as the correlation functions involving these fields depend only on its homotopy properties. The field (2.2) creates a discontinuity of the field $\varphi$ along $\mathcal{C}_{x}$, so that the values of $\varphi$ right across this contour differ by a constant equal $2 \pi n / \beta$. In operator formalism, the field operator associated with (2.2) creates the topological charge $n$. The fields (2.2) are mutually local and they have zero spin. Since the exponential in the r.h.s. of (2.2) requires regularization this expression defines the field $\mathcal{O}_{0}^{n}$ only up to normalization. We fix this ambiguity by assuming the "CFT normalization" (1.1), specifically

$$
\lim _{|x| \rightarrow 0}|x|^{\frac{n^{2}}{8 \beta^{2}}}\left\langle\mathcal{O}_{0}^{n}(x) \mathcal{O}_{0}^{n}(0)\right\rangle=1
$$

Although the fields (2.2) are themselves local, they obviously are not local with respect to the field $\varphi(x)$. Nevertheless one can define a class of useful nonlocal fields $\mathcal{O}_{a}^{n}(x)$ by "fusing" the fields (2.2) with the exponentials of $\varphi$, i.e. by taking the limits $\lim _{x^{\prime} \rightarrow x} \mathcal{O}_{0}^{n}(x) \exp \left(i a \varphi\left(x^{\prime}\right)\right)$. In view of the above nonlocality this limit has a phase ambiguity, which can be fixed, for instance, by specifying the direction, relative to the contour $\mathcal{C}_{x}$, from which $x^{\prime}$ approaches $x$. To eliminate all ambiguities one can choose some cartesian coordinates $x=(\mathrm{x}, \mathrm{y})$, and define

$$
\mathcal{O}_{a}^{n}(x)=\lim _{\epsilon \rightarrow+0} \exp \left\{-\frac{n}{4 \beta} \int_{-\infty}^{\mathrm{x}} \partial_{\mathrm{y}} \varphi(\mathrm{x}, \mathrm{y}) d \mathrm{x}\right\} \exp \{i a \varphi(\mathrm{x}+\epsilon, \mathrm{y})\} .
$$

1 Here and below we use the notations and conventions adopted in [4]; in particular, the language of euclidean field theory is used by default. 
In fact, rigid specification of the contour $\mathcal{C}_{x}$ in (2.4) results in inconveniently discontinuous correlation functions involving these objects. To avoid this inessential complication, in what follows by a correlation function

$$
\left\langle\mathcal{O}_{a_{N}}^{n_{N}}\left(\mathrm{x}_{N}, \mathrm{y}_{N}\right) \cdots \mathcal{O}_{a_{1}}^{n_{1}}\left(\mathrm{x}_{1}, \mathrm{y}_{1}\right)\right\rangle
$$

we always understand the analytic continuation of this correlator from the domain $\mathrm{y}_{1}<\mathrm{y}_{2}<\cdots<\mathrm{y}_{N}$. With this definition the correlation function (2.5) becomes a multivalued function of the coordinates $x_{1}, \cdots, x_{N}$ which acquires a certain phase factor $\exp \left(-i \pi\left(a_{j} n_{k}+a_{k} n_{j}\right) / \beta\right)$ when the point $x_{j}$ is brought around $x_{k}$ counterclockwise. In particular, the two-point correlation function can be written as

$$
\left\langle\mathcal{O}_{a}^{n}(x) \mathcal{O}_{a^{\prime}}^{-n}(0)\right\rangle=\left(e^{i \pi} \frac{\overline{\mathrm{Z}}}{\mathrm{Z}}\right)^{\frac{s\left(a^{\prime}, n\right)-s(a, n)}{2}} e^{i \pi \frac{s(a, n)+s\left(a^{\prime}, n\right)}{2}} \mathcal{G}_{a, a^{\prime}}^{(n)}(r)
$$

where

$$
s(a, n)=\frac{a n}{\beta},
$$

$\mathrm{z}=\mathrm{x}+i \mathrm{y}, \overline{\mathrm{z}}=\mathrm{x}-i \mathrm{y}$, and the function $\mathcal{G}_{a, a^{\prime}}$ is real and depends only on the distance $r=\sqrt{\mathrm{z} \overline{\mathrm{z}}}$. In the terminology of [10] the fields $\mathcal{O}_{a}^{n}$ are "semi-local". If $a \neq 0$ the field $\mathcal{O}_{a}^{n}$ carries nonzero spin (2.7). It becomes a local, bosonic or fermionic, field when $s(a, n)$ takes integer or half-integer values. Note that the definition (2.4) fixes also the normalization of the field $\mathcal{O}_{a}^{n}$, since the normalizations of $\mathcal{O}_{0}^{n}(x)$ and $\mathcal{O}_{a}^{0}(x) \equiv e^{i a \varphi}$ are already fixed through (2.3) and (1.1), respectively. The same normalization can be specified by the condition

$$
\left.\mathcal{G}_{a,-a}^{(n)}(r)\right|_{r \rightarrow 0} \rightarrow \frac{1}{r^{2 d(a, n)}} \quad \text { with } \quad d(a, n)=2 a^{2}+\frac{n^{2}}{8 \beta^{2}}
$$

The aim of this work is to describe the form factors of the operators $\mathcal{O}_{a}^{n}$ with $n$-soliton states. We will assume the hamiltonian picture with coordinate y taken as euclidean time. Note that in this picture the operators defined by (2.4) obey the simple hermiticity relation

$$
\left(\mathcal{O}_{a}^{n}\right)^{\dagger}=\mathcal{O}_{-a}^{-n}
$$

which allows us to restrict our attention to the form factors of $\mathcal{O}_{a}^{n}$ with generic $a$ but $n \geq 0$. We will use the notation $A_{-}$and $A_{+}$for the soliton and antisoliton; these particles carry negative and positive units of the topological charge,

$$
H=\frac{\beta}{2 \pi} \int_{-\infty}^{\infty} \partial_{\mathrm{x}} \varphi(\mathrm{x}, \mathrm{y}) d \mathrm{x}
$$


respectively. Conservation of the topological charge implies that nonvanishing form factors of the operator $\mathcal{O}_{a}^{n}$ are of the form

$$
\left\langle v a c\left|\mathcal{O}_{a}^{n}(0)\right| A_{-}\left(\theta_{1}\right) \cdots A_{-}\left(\theta_{n+N}\right) A_{+}\left(\theta_{1}^{\prime}\right) \cdots A_{+}\left(\theta_{N}^{\prime}\right)\right\rangle
$$

where $\theta_{i}$ and $\theta_{j}^{\prime}$ denote rapidities of solitons and antisolitons (for simplicity, here and below we ignore the possible presence of breathers). Up to overall normalization, all these form factors can be written down in closed form, as certain $N$-fold integrals [8]. In the simplest case, $N=0$, an explicit formula exists,

$$
\left\langle v a c\left|\mathcal{O}_{a}^{n}(0)\right| A_{-}\left(\theta_{1}\right) \cdots A_{-}\left(\theta_{n}\right)\right\rangle_{i n}=\sqrt{\mathbf{Z}_{n}(a)} e^{\frac{i \pi n a}{2 \beta}} \prod_{m=1}^{n} e^{\frac{a}{\beta} \theta_{m}} \prod_{m<j}^{n} G\left(\theta_{m}-\theta_{j}\right)
$$

Here

$$
G(\theta)=i \mathcal{C}_{1} \sinh (\theta / 2) \exp \left(\int_{0}^{\infty} \frac{d t}{t} \frac{\sinh ^{2} t(1-i \theta / \pi) \sinh (t(\xi-1))}{\sinh (2 t) \sinh (t \xi) \cosh (t)}\right)
$$

with

$$
\mathcal{C}_{1}=\exp \left(-\int_{0}^{\infty} \frac{d t}{t} \frac{\sinh ^{2}(t / 2) \sinh (t(\xi-1))}{\sinh (2 t) \sinh (t \xi) \cosh (t)}\right)
$$

and we have assumed that the rapidities are arranged so that $\theta_{1}<\theta_{2}<\cdots<\theta_{n}$. Here and below we use the notation

$$
\xi=\frac{\beta^{2}}{1-\beta^{2}}
$$

The only unknown component in (2.12) is the real normalization constant $\mathbf{Z}_{n}(a)$. This constant controls the long-distance asymptotics of the two-point function (2.6) (with the normalization already fixed by (2.8)) because it is dominated by $n$-soliton intermediate states, namely

$$
\mathcal{G}_{a, a^{\prime}}^{(n)}(r)=\frac{\sqrt{\mathbf{Z}_{n}(a) \mathbf{Z}_{n}\left(a^{\prime}\right)}}{n !} \int_{-\infty}^{+\infty} \prod_{m<j}\left|G\left(\theta_{m}-\theta_{j}\right)\right|^{2} \prod_{m=1}^{n} e^{\frac{\left(a-a^{\prime}\right)}{\beta} \theta_{m}-M r \cosh \theta_{m}} \frac{d \theta_{m}}{2 \pi}+\ldots
$$

where $M$ is the soliton mass, and the dots stand for the subleading terms, which are of the order of $e^{-(n+2) M r}$. Let us also stress here that once the constant $\mathbf{Z}_{n}(a)$ in (2.12) is fixed, the normalizations of all higher form factors (2.11) are also fixed by the "annihilation pole" condition of the form factor bootstrap (see [1] for details). For example, using the 
approach of [8], one can derive the following expression for the $n+2$-particle form factor (2.11),

$$
\begin{aligned}
& \left\langle\text { vac }\left|\mathcal{O}_{a}^{n}(0)\right| A_{-}\left(\theta_{1}\right) \ldots A_{+}\left(\theta_{k}\right) \ldots A_{-}\left(\theta_{n+2}\right)\right\rangle_{i n}=\frac{i \mathcal{C}_{2} \sqrt{\mathbf{Z}_{n}(a)}}{4 \mathcal{C}_{1}} e^{\frac{i \pi a n}{2 \beta}} \prod_{m=1}^{n+2} e^{\frac{a}{\beta} \theta_{m}} \times \\
& e^{\frac{\theta_{k}}{\xi}} \prod_{m<j} G\left(\theta_{m}-\theta_{j}\right)\left\{e^{\frac{i \pi}{2 \beta^{2}}} \int_{C_{+}} \frac{d \gamma}{2 \pi} e^{-\left(\frac{2 a}{\beta}+\frac{1}{\xi}\right) \gamma} \prod_{p=1}^{k} W\left(\theta_{p}-\gamma\right) \prod_{p=k+1}^{n+2} W\left(\gamma-\theta_{p}\right)-\right. \\
& \left.e^{-\frac{i \pi}{2 \beta^{2}}} \int_{C_{-}} \frac{d \gamma}{2 \pi} e^{-\left(\frac{2 a}{\beta}+\frac{1}{\xi}\right) \gamma} \prod_{p=1}^{k-1} W\left(\theta_{p}-\gamma\right) \prod_{p=k}^{n+2} W\left(\gamma-\theta_{p}\right)\right\} .
\end{aligned}
$$

Here the function $W$ and the constant $\mathcal{C}_{2}$ are

$$
\begin{aligned}
& W(\theta)=-\frac{2}{\cosh (\theta)} \exp \left(-2 \int_{0}^{\infty} \frac{d t}{t} \frac{\sinh ^{2} t(1-i \theta / \pi) \sinh (t(\xi-1))}{\sinh (2 t) \sinh (t \xi)}\right) \\
& \mathcal{C}_{2}=\exp \left(4 \int_{0}^{\infty} \frac{d t}{t} \frac{\sinh ^{2}(t / 2) \sinh (t(\xi-1))}{\sinh (2 t) \sinh (t \xi)}\right) .
\end{aligned}
$$

The integration contours $C_{+}$and $C_{-}$in (2.17) are described as follows. The contour $C_{+}$ starts from $-\infty$ along the real axis of the complex $\gamma$ plane, and winds around the poles of its integrand located in the strip $-\frac{\pi}{2}-0<\Im m \gamma<\frac{\pi}{2}+0$, going first above the poles at $\theta_{p}+\frac{i \pi}{2}, \quad p=1, \ldots k$, and then below the poles at $\theta_{p}-\frac{i \pi}{2}, \quad p=k+1, \ldots n+2$, and finally extends to $+\infty$ along the real axis, as illustrated in Fig.1a. Similarly, the contour $C_{-}$goes above $\theta_{p}+\frac{i \pi}{2}, \quad p=1, \ldots k-1$ and below $\theta_{p}-\frac{i \pi}{2}, \quad p=k, \ldots n+2$ of its integrand, see Fig.1b.

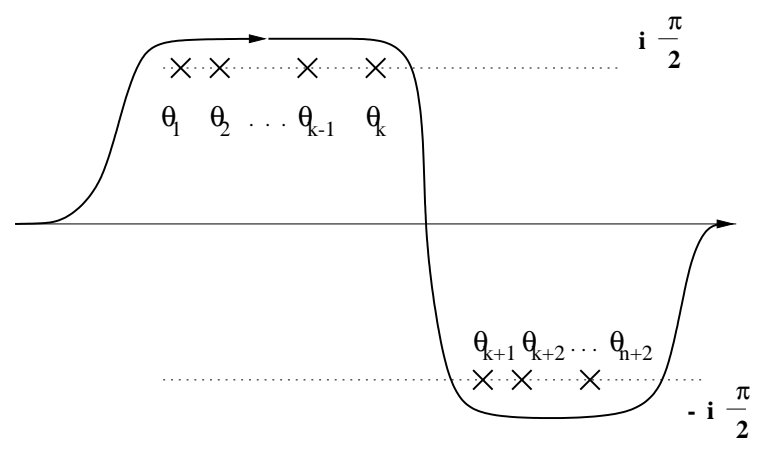

Fig.1a

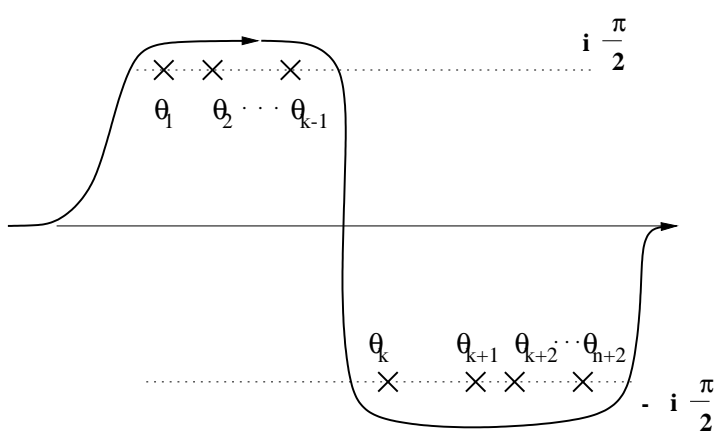

Fig.1b 
Under the assumed normalization of the fields $\mathcal{O}_{a}^{n}$, the leading short-distance asymptotic of the two-point correlation function (2.6) is

$$
\left.\mathcal{G}_{a, a^{\prime}}^{(n)}(r)\right|_{r \rightarrow 0} \rightarrow \frac{\left\langle e^{i\left(a+a^{\prime}\right) \varphi}\right\rangle}{r^{d(a, n)+d\left(a^{\prime}, n\right)-d\left(a+a^{\prime}, 0\right)}},
$$

with the coefficient $\mathcal{G}_{a+a^{\prime}} \equiv\left\langle e^{i\left(a+a^{\prime}\right) \varphi}\right\rangle$ which can be read off from $\llbracket 4$. To determine the coefficient in its leading large-distance asymptotic (2.16) one needs to know the normalization constant $\mathbf{Z}_{n}(a)$ in (2.12).

\section{Conjecture}

We propose the following explicit formula for the constant $\mathbf{Z}_{n}(a)$ in (2.12):

$$
\begin{aligned}
& \sqrt{\mathbf{Z}_{n}(a)}=\left(\frac{\mathcal{C}_{2}}{2 \mathcal{C}_{1}^{2}}\right)^{\frac{n}{4}}\left(\frac{\xi \mathcal{C}_{2}}{16}\right)^{-\frac{n^{2}}{8}}\left[\frac{\sqrt{\pi} M \Gamma\left(\frac{3}{2}+\frac{\xi}{2}\right)}{\Gamma\left(\frac{\xi}{2}\right)}\right]^{d(a, n)} \times \\
& \exp \left[\int_{0}^{\infty} \frac{d t}{t}\left\{\frac{\cosh (4 \xi a t / \beta) e^{-(1+\xi) n t}-1}{4 \sinh (\xi t) \sinh ((1+\xi) t) \cosh (t)}+\frac{n}{4 \sinh (t \xi)}-d(a, n) e^{-2 t}\right\}\right] .
\end{aligned}
$$

Here the constants $\mathcal{C}_{1}$ and $\mathcal{C}_{2}$ are given by (2.14) and (2.18), and $d(a, n)$ is as in (2.8). We would like to stress here that this formula should be used only with $n \geq 0$. For example, the form factor $\left\langle v a c\left|\mathcal{O}_{a}^{-n}(0)\right| A_{+}\left(\theta_{1}\right) \cdots A_{+}\left(\theta_{n}\right)\right\rangle_{\text {in }}$ should be written with $\sqrt{\mathbf{Z}_{n}(a)}$ (not $\left.\sqrt{\mathbf{Z}_{-n}(a)}\right)$ for its normalization constant.

In the next section some calculations supporting the conjecture (3.1) are presented (and some are just mentioned).

\section{Supporting calculations}

\subsection{The case $n=0$}

In this case the field (2.4) reduces to the exponential field $\exp (i a \varphi(x))$, and the form factor (2.12) becomes its vacuum-vacuum matrix element. Correspondingly, for $n=0$ (3.1) reduces to the expectation value of this exponential field (see 四).

\subsection{Free fermion point}

At $\beta^{2}=1 / 2$ the sine-Gordon model reduces to free Dirac fermions. In this case the form factors (2.12), and in particular the normalization factors $\mathbf{Z}_{n}(a)$, can be calculated directly [11]. For this value of $\beta^{2}$ Eq.(3.1) coincides with the result of [11]. 


\subsection{Massive Thirring Model perturbation theory}

As is well known [12,13 the sine-Gordon theory is equivalent to the massive Thirring model,

$$
\mathcal{A}_{M T M}=\int d^{2} x\left\{\bar{\Psi} \gamma_{\mu} \partial_{\mu} \Psi+\mathcal{M} \bar{\Psi} \Psi+\frac{g}{2} J_{\mu} J_{\mu}\right\}
$$

where $\Psi$ is a Dirac Fermi field, $J_{\mu}=\bar{\Psi} \gamma_{\mu} \Psi$ is its (non-anomalous) vector current, and the coupling constant $g$ is related to $\beta$ in (2.1) by

$$
\frac{g}{\pi}=\frac{1}{2 \beta^{2}}-1
$$

The action (4.1) requires field and mass-term renormalizations, and the precise relation between $\mathcal{M}$ and $\mu$ in (2.1) depends on the renormalization scheme. The fields $\Psi, \bar{\Psi}$ are related to $\mathcal{O}_{ \pm \beta / 2}^{ \pm 1}$ from Sect.2 as follows 13

$$
\Psi(x)=\frac{1}{\sqrt{2 \pi \mathbf{Z}_{\Psi}}}\left(\begin{array}{c}
\mathcal{O}_{-\beta / 2}^{1} \\
\mathcal{O}_{\beta / 2}^{1}
\end{array}\right), \quad \bar{\Psi}(x)=\frac{1}{\sqrt{2 \pi \mathbf{Z}_{\Psi}}}\left(\mathcal{O}_{-\beta / 2}^{-1}, \mathcal{O}_{\beta / 2}^{-1}\right)
$$

where we have chosen a chiral representation of the (euclidean) Dirac matrices, i.e. $\gamma_{\mathrm{x}}=$ $-\sigma_{2}, \gamma_{\mathrm{y}}=\sigma_{1}$. The constant $\mathbf{Z}_{\Psi}$ depends on the normalization condition for the field $\Psi$. In what follows we assume the most common renormalization scheme, in which the renormalized $\mathcal{M}$ coincides with the soliton mass $M$, and the normalization of $\Psi$ is fixed as follows,

$$
(i \not p+M) S(p) \rightarrow 1 \quad \text { as } \quad p^{2}+M^{2} \rightarrow 0
$$

Here $S(p)$ is the momentum-space propagator,

$$
\langle\Psi(x) \bar{\Psi}(0)\rangle=\int S(p) e^{i p x} \frac{d^{2} p}{(2 \pi)^{2}}
$$

and $\not p$ stands for $\gamma_{\mu} p_{\mu}$. Comparing this equation with the $n=1$ and $a=-a^{\prime}=-\beta / 2$ case of (2.16), one finds that this scheme corresponds to the choice $\mathbf{Z}_{\Psi}=\mathbf{Z}_{1}(\beta / 2)$ in (4.3). On the other hand, the short-distance behavior of the correlation functions $\left\langle\mathcal{O}_{-\beta / 2}^{1} \mathcal{O}_{\beta / 2}^{-1}\right\rangle$ is given by $(2.6),(2.8)$. This leads to the following prediction for the constant factor in the large-momentum asymptotic of the fermion propagator in this scheme,

$$
S(p) \rightarrow-i \not p \quad \frac{M \pi}{\mathbf{Z}_{\Psi}} \frac{2^{1-2 d_{\Psi}} \Gamma\left(3 / 2-d_{\Psi}\right)}{\Gamma\left(1 / 2+d_{\Psi}\right)} \quad\left(p^{2}\right)^{d_{\Psi}-\frac{3}{2}} \quad \text { as } \quad p^{2} \rightarrow \infty
$$


Here

$$
d_{\Psi}=\frac{1}{8 \beta^{2}}+\frac{\beta^{2}}{2}
$$

and

$$
\begin{aligned}
& \mathbf{Z}_{\Psi}=\left(\frac{\mathcal{C}_{2}}{2 \mathcal{C}_{1}^{2}}\right)^{\frac{1}{2}}\left(\frac{\xi \mathcal{C}_{2}}{16}\right)^{-\frac{1}{4}}\left[\frac{\sqrt{\pi} M \Gamma\left(\frac{3}{2}+\frac{\xi}{2}\right)}{\Gamma\left(\frac{\xi}{2}\right)}\right]^{2 d_{\Psi}} \times \\
& \exp \left[\int_{0}^{\infty} \frac{d t}{t}\left\{\frac{\cosh (2 \xi t) e^{-(1+\xi) t}-1}{2 \sinh (\xi t) \sinh ((1+\xi) t) \cosh (t)}+\frac{1}{2 \sinh (t \xi)}-2 d_{\Psi} e^{-2 t}\right\}\right] .
\end{aligned}
$$

This prediction can be verified in standard renormalized perturbation theory in (4.1). Straightforward evaluation of the diagram in Fig.2 yields

$$
S^{-1}(p)=i \not p+M+\left(\frac{g}{2 \pi}\right)^{2} \Sigma_{2}(p)+O\left(g^{3}\right),
$$

where

$$
\Sigma_{2}(p) \rightarrow-i \not p\left\{\frac{\pi^{2}}{3}-6+\log \left(\frac{p^{2}}{M^{2}}\right)\right\} \quad \text { as } \quad p^{2} \rightarrow \infty .
$$

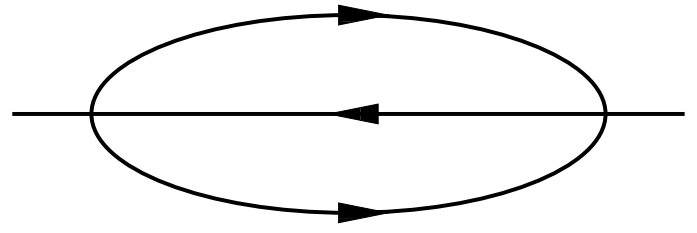

Fig.2

Using a power expansion of (4.5) in the parameter (4.2),

$$
\mathbf{Z}_{\Psi}=\pi M\left\{1+\left(\frac{g}{2 \pi}\right)^{2}\left(\log \left(M^{2}\right)+2 \gamma_{E}+6-2 \log 2-\frac{\pi^{2}}{3}\right)+O\left(g^{3}\right)\right\},
$$

where $\gamma_{E}=0.577216 \ldots$ is Euler's constant, one can check that (4.4) agrees with (4.6) to order $g^{2}$.

\subsection{Nonlocal Integrals of Motion}

As was mentioned in the Introduction, the fields $\mathcal{O}_{a}^{ \pm 2}$ with appropriately chosen values of $a$ coincide with the components of the nonlocal currents found in [14]. Namely, the fields

$$
\begin{aligned}
\mathcal{J}_{ \pm}(x)=\mathcal{O}_{ \pm(2 \beta)^{-1}}^{ \pm 2}(x), & \mathcal{H}_{ \pm}(x)=\pi \xi \mu \mathcal{O}_{ \pm \nu}^{ \pm 2}(x), \\
\overline{\mathcal{J}}_{ \pm}(x)=\mathcal{O}_{\mp(2 \beta)^{-1}}^{ \pm 2}(x), & \overline{\mathcal{H}}_{ \pm}(x)=\pi \xi \mu \mathcal{O}_{\mp \nu}^{ \pm 2}(x),
\end{aligned}
$$


with $\nu=(2 \beta)^{-1}-\beta$, satisfy the continuity equations

$$
\bar{\partial} \mathcal{J}_{ \pm}(x)=\partial \mathcal{H}_{ \pm}, \quad \partial \mathcal{J}_{ \pm}(x)=\bar{\partial} \mathcal{H}_{ \pm}
$$

where $\partial=\frac{1}{2}\left(\partial_{\mathrm{x}}-i \partial_{\mathrm{y}}\right), \quad \bar{\partial}=\frac{1}{2}\left(\partial_{\mathrm{x}}+i \partial_{\mathrm{y}}\right)$. They give rise to four nonlocal integrals of motion

$$
\begin{aligned}
& Q_{ \pm}=\frac{1}{\mathbf{Z}_{Q}} \int_{-\infty}^{\infty}\left(\mathcal{J}_{ \pm}(\mathrm{x}, \mathrm{y})+\mathcal{H}_{ \pm}(\mathrm{x}, \mathrm{y})\right) d \mathrm{x} \\
& \bar{Q}_{ \pm}=\frac{1}{\mathbf{Z}_{Q}} \int_{-\infty}^{\infty}\left(\overline{\mathcal{J}}_{ \pm}(\mathrm{x}, \mathrm{y})+\overline{\mathcal{H}}_{ \pm}(\mathrm{x}, \mathrm{y})\right) d \mathrm{x}
\end{aligned}
$$

which generate (as was found in [14]) the affine quantum group $U_{q}(\widehat{s l(2)})$ of level zero, with

$$
q=e^{i \pi / \beta^{2}}
$$

More precisely, under a special choice of the ( $\beta$ - and $\mu$-dependent) constant $\mathbf{Z}_{Q}$, the operators $(4.10)$ obey the commutation relations

$$
Q_{-} \bar{Q}_{+}-q^{2} \bar{Q}_{+} Q_{-}=\frac{1-q^{2 H}}{1-q^{-2}}, \quad Q_{+} \bar{Q}_{-}-q^{2} \bar{Q}_{-} Q_{+}=\frac{1-q^{-2 H}}{1-q^{-2}}
$$

where $H$ is the topological charge (2.10). The constant $\mathbf{Z}_{Q}$ can be expressed through the expectation value of the fields $e^{i(\beta-1 / \beta) \varphi}$,

$$
\mathbf{Z}_{Q}^{2}=\mu \xi(1+\xi) \sin (\pi / \xi)\left\langle e^{i(\beta-1 / \beta) \varphi}\right\rangle=\frac{1}{\Gamma^{2}(1+1 / \xi)}\left[\frac{\sqrt{\pi} M \Gamma\left(\frac{3}{2}+\frac{\xi}{2}\right)}{2 \Gamma\left(1+\frac{\xi}{2}\right)}\right]^{\frac{2}{\xi}}
$$

where again $M$ denotes the soliton mass.

The vacuum $|v a c\rangle$ is annihilated by all the generators (4.10), while the solitonantisoliton pair $A_{ \pm}$forms two-dimensional representation of the algebra (4.11),

$$
\begin{aligned}
& Q_{ \pm}\left|A_{ \pm}(\theta)\right\rangle=\bar{Q}_{ \pm}\left|A_{ \pm}(\theta)\right\rangle=0, \\
& Q_{\mp}\left|A_{ \pm}(\theta)\right\rangle=e^{\frac{\theta}{\xi}}\left|A_{\mp}(\theta)\right\rangle, \quad \bar{Q}_{\mp}\left|A_{ \pm}(\theta)\right\rangle=e^{-\frac{\theta}{\xi}}\left|A_{\mp}(\theta)\right\rangle,
\end{aligned}
$$

and $H\left|A_{ \pm}(\theta)\right\rangle= \pm\left|A_{ \pm}(\theta)\right\rangle$. The action of these operators on any multisoliton state is described by a standard $U_{q}(\widehat{s l(2)})$ coproduct, i.e.

$$
\Delta\left(Q_{ \pm}\right)=Q_{ \pm} \otimes 1+q^{\mp H} \otimes Q_{ \pm}, \quad \Delta\left(\bar{Q}_{ \pm}\right)=\bar{Q}_{ \pm} \otimes 1+q^{ \pm H} \otimes \bar{Q}_{ \pm}
$$


It is not difficult to see that consistency of the form factor formula (2.12) with the above continuity equation (4.9) and with (4.13) requires that the coefficient $\mathbf{Z}_{n}(a)$ in (2.12) satisfy the following identities

$$
\mathbf{Z}_{2}\left(\frac{1}{2 \beta}\right)=(\pi \xi \mu)^{2} \mathbf{Z}_{2}\left(\frac{1}{2 \beta}-\beta\right)=\left(\frac{M \mathbf{Z}_{Q}}{2 \mathcal{C}_{1}}\right)^{2}
$$

which both are easily verified for (3.1).

More interesting consistency conditions come from the following observation. For all integer $n$ and $m$ the fields $\mathcal{O}_{a_{n, m}}^{n}$ with

$$
a_{n, m}=\frac{n}{4 \beta}+\frac{m \beta}{2}
$$

are local with respect the currents $\overline{\mathcal{J}}_{ \pm}$and $\overline{\mathcal{H}}_{ \pm}$defined in (4.8) (a similar set $\mathcal{O}_{-a_{n, m}}^{n}$ exists for the currents $\mathcal{J}_{ \pm}$and $\mathcal{H}_{ \pm}$). As a result, commutators (more precisely, " $q$-commutators") of $\mathcal{O}_{a_{n, m}}^{n}$ with the generators $\bar{Q}_{+}$and $\bar{Q}_{-}$are expressed through the fields $\mathcal{O}_{a}^{n \pm 2}$ (with the parameter $a$ somewhat shifted) or their descendants. In particular, one can derive the following commutation relation

$$
\bar{Q}_{+} \mathcal{O}_{a_{n, 1}}^{n}(x)-q^{n} \mathcal{O}_{a_{n, 1}}^{n}(x) \bar{Q}_{+}=\frac{2 \pi i}{\mathbf{Z}_{Q}} q^{\frac{n}{2}} \mathcal{O}_{a_{n-2,1}}^{n+2}(x)
$$

This operator equation of course leads to certain relations between the form factors. For instance, sandwiching (4.15) between the vacuum and the state containing $n+2$ antisolitons and using (4.14) and (4.13) to transform the matrix element appearing on the left-hand side, one obtains

$$
\begin{aligned}
\sum_{k=1}^{n+2} q^{k-n-2} & e^{-\frac{\theta_{k}}{\xi}}\left\langle\operatorname{vac}\left|\mathcal{O}_{a_{n, 1}}^{n}(0)\right| A_{-}\left(\theta_{1}\right) \cdots A_{+}\left(\theta_{k}\right) \cdots A_{-}\left(\theta_{n+2}\right)\right\rangle_{i n}= \\
& -\frac{2 \pi i}{\mathbf{Z}_{Q}} q^{-\frac{n}{2}}\left\langle\operatorname{vac}\left|\mathcal{O}_{a_{n-2,1}}^{n+2}(0)\right| A_{-}\left(\theta_{1}\right) \cdots A_{-}\left(\theta_{n+2}\right)\right\rangle_{i n} .
\end{aligned}
$$

Now, substituting (2.17) for the form factors in the left-hand side of (4.16), one observes that all but two integrals in the sum cancel each other, which results in the relation

$$
\begin{aligned}
& \frac{2 \pi}{\mathbf{Z}_{q}} \sqrt{\frac{\mathbf{Z}_{n+2}\left(a_{n-2,1}\right)}{\mathbf{Z}_{n}\left(a_{n, 1}\right)}}=\frac{i \mathcal{C}_{2}}{4 \mathcal{C}_{1}} \prod_{k=1}^{n+2} e^{\frac{\theta_{k}}{2 \beta^{2}}} \times \\
& \left\{q^{\frac{n}{2}+1} \int_{C_{+}} \frac{d \gamma}{2 \pi} e^{-\frac{\gamma(n+2)}{2 \beta^{2}}} \prod_{k=1}^{n+2} W\left(\theta_{k}-\gamma\right)-q^{-\frac{n}{2}-1} \int_{C_{-}} \frac{d \gamma}{2 \pi} e^{-\frac{\gamma(n+2)}{2 \beta^{2}}} \prod_{k=1}^{n+2} W\left(\gamma-\theta_{k}\right)\right\},
\end{aligned}
$$


where $W$ is the function (2.18). Thanks to the identity $W(\theta-i \pi)=W(-\theta-i \pi)$, the sum of the integrals in the bracket $\{\cdots\}$ on the r.h.s. of (4.17) in fact reduces to the following limit

$$
\{\cdots\}=\lim _{\Lambda \rightarrow+\infty} \int_{-\Lambda-i \pi}^{-\Lambda+i \pi} \frac{d \gamma}{2 \pi} e^{-\frac{\gamma(n+2)}{2 \beta^{2}}} \prod_{k=1}^{n+2} W\left(\theta_{k}-\gamma-i \pi\right)=i\left(\frac{\mathcal{C}_{2} \xi}{16}\right)^{-\frac{n}{2}-1} \prod_{k=1}^{n+2} e^{-\frac{\theta_{k}}{2 \beta^{2}}} .
$$

In turn, the limit can be evaluated using the asymptotic behavior

$$
W(\theta-i \pi) \rightarrow\left(\frac{\mathcal{C}_{2} \xi}{16}\right)^{-\frac{1}{2}} e^{-\frac{\theta}{2 \beta^{2}}} \quad \text { as } \quad \Re e \theta \rightarrow+\infty,
$$

and therefore (4.15) leads to the following relation between the normalization factors in $(2.12)$ :

$$
\frac{\mathbf{Z}_{n+2}\left(a_{n-2,1}\right)}{\mathbf{Z}_{n}\left(a_{n, 1}\right)}=\left(\frac{2 \mathbf{Z}_{Q}}{\pi \xi \mathcal{C}_{1}}\right)^{2}\left(\frac{\mathcal{C}_{2} \xi}{16}\right)^{-n}
$$

It is possible to check that (3.1) indeed obeys (4.18).

\subsection{Scattering in the lattice $X Y Z$ model}

The sine-Gordon field theory describes the scaling limit of the integrable $X Y Z$ spin chain [15],

$$
\mathbf{H}_{X Y Z}=-\frac{1}{2} \sum_{k=1}^{N}\left(J_{x} \sigma_{k}^{x} \sigma_{k+1}^{x}+J_{y} \sigma_{k}^{y} \sigma_{k+1}^{y}+J_{z} \sigma_{k}^{z} \sigma_{k+1}^{z}\right)
$$

As is known, at $J_{y}=J_{x} \geq\left|J_{z}\right|$ the spin chain (4.19) is critical, i.e. the gap in the spectrum of (4.19) vanishes, and its correlation length $R_{c}$ becomes infinite in units of the lattice spacing [16. In the scaling limit $J_{x}-J_{y} \rightarrow 0$ the spin correlations in (4.19) are described by the field theory (2.1) with [17,16]

$$
\begin{aligned}
& \cos \left(\pi \beta^{2}\right)=J_{z} / J_{x}, \\
& \left(\frac{M \varepsilon}{4}\right)^{2-2 \beta^{2}} \simeq \frac{\left|J_{x}-J_{y}\right|}{8 \sin ^{2}\left(\pi \beta^{2}\right) J_{x}} \rightarrow 0,
\end{aligned}
$$

provided all relevant distances are $\sim R_{c}$, i.e. infinitely greater than the lattice spacing $\varepsilon$. If, however, $J_{x}-J_{y}$ is small but finite, there are corrections due to finite lattice size. These corrections can be taken into account by adding certain irrelevant perturbations to (2.1), i.e. by using the "effective action"

$$
\mathcal{A}_{e f f}=\mathcal{A}_{s g}+\int \frac{d^{2} x}{2 \pi}\left\{\frac{\lambda}{2}\left(\mathcal{O}_{0}^{4}(x)+\mathcal{O}_{0}^{-4}(x)\right) \varepsilon^{\frac{2}{\xi}}-\left(\lambda_{+} \mathbf{T} \overline{\mathbf{T}}+\lambda_{-}\left(\mathbf{T}^{2}+\overline{\mathbf{T}}^{2}\right)\right) \varepsilon^{2}+\ldots\right\}
$$


where we have written down explicitly only the most significant of those irrelevant terms. Here $\mathbf{T}$ and $\overline{\mathbf{T}}$ are components of the sine-Gordon stress-energy tensor, in standard notations, and $\mathcal{O}_{0}^{ \pm 4}(x)$ are the fields (2.2). In (4.20) the explicit dependence on the lattice spacing $\varepsilon$ is exhibited to show the relative smallness of various terms; the omitted terms are of higher order in $\varepsilon$. Fortunately, the dimensionless coupling constants $\lambda_{ \pm}$and $\lambda$ in (4.20) are known exactly [18], in particular

$$
\lambda=\frac{4 \Gamma(1+1 / \xi)}{\Gamma(-1 / \xi)}\left(\frac{\Gamma\left(1+\frac{\xi}{2}\right)}{2 \sqrt{\pi} \Gamma\left(\frac{3}{2}+\frac{\xi}{2}\right)}\right)^{\frac{2}{\xi}} .
$$

This makes (4.20) a working tool for determining the leading lattice corrections to the scaling limit in the $X Y Z$ model.

Since the fields $\mathcal{O}_{0}^{ \pm 4}$ carry nonzero topological charge, the $\lambda$-terms in (4.20) generate processes violating conservation of the topological charge, which is now conserved only modulo 4. In particular, two solitons can be produced in antisoliton-antisoliton scattering, with nonzero amplitude. In the Born approximation this amplitude is

$$
S_{--}^{++}\left(\theta_{2}-\theta_{1}\right)=\frac{(-i) \lambda \varepsilon^{\frac{2}{\xi}}}{4 \pi M^{2} \sinh \left(\theta_{2}-\theta_{1}\right)} \quad \text { out }\left\langle A_{+}\left(\theta_{1}\right) A_{+}\left(\theta_{2}\right)\left|\mathcal{O}_{0}^{4}(0)\right| A_{-}\left(\theta_{1}\right) A_{-}\left(\theta_{2}\right)\right\rangle_{\text {in }}
$$

where $\theta_{1}$ and $\theta_{2}\left(\theta_{1}<\theta_{2}\right)$ are rapidities describing the kinematics of the scattering. The matrix element here can be obtained by crossing transformation from the form factor (3.1) with $n=4$ and $a=0$. Simple calculation yields

$$
S_{--}^{++}(\theta)=i K \sinh (\theta / \xi) S(\theta)
$$

where

$$
S(\theta)=G(-\theta) / G(\theta)
$$

is the sine-Gordon $S$-matrix element associated with elastic process $A_{-} A_{-} \rightarrow A_{-} A_{-}$, and

$$
K=-\frac{\xi \mathcal{C}_{1}^{2} \mathcal{C}_{2} \lambda \varepsilon^{\frac{2}{\xi}}}{16 \pi M^{2}} \sqrt{\mathbf{Z}_{4}(0)}
$$

The appearance of this amplitude is of course expected. The scattering of excitations in the lattice model (4.19) is described by Baxter's elliptic S-matrix. It depends on the elliptic nome $\tilde{p}$, whose precise relation to the parameters in (4.19) is rather transcendental (one 
can consult [19,20] for details), but here we only need to know that when $\left|J_{x}-J_{y}\right| \rightarrow 0$ this nome also goes to zero as

$$
\tilde{p} \simeq\left(\frac{M \varepsilon}{4}\right)^{\frac{2}{\xi}}
$$

If $J_{x}-J_{y} \neq 0$ this elliptic S-matrix has a nonzero element $S_{--}^{++}(\theta)$, presented explicitly in 19,20. One can check that when $\tilde{p}$ gets small this amplitude assumes precisely the form (4.21) with

$$
K=4 \tilde{p} \sin (\pi / \xi)
$$

This agrees with (4.22) provided

$$
\sqrt{\mathbf{Z}_{4}(0)}=\frac{M^{2}}{\mathcal{C}_{1}^{2} \mathcal{C}_{2}} \frac{16 \pi^{2} \xi}{\Gamma^{2}(1 / \xi)}\left(\frac{M \sqrt{\pi} \Gamma\left(\frac{3}{2}+\frac{\xi}{2}\right)}{2 \Gamma\left(1+\frac{\xi}{2}\right)}\right)^{\frac{2}{\xi}}
$$

It is not difficult to verify that for given values of the parameters (3.1) indeed reduces to (4.24).

\section{Deformed $S U(2)$-Thirring model}

The normalization factor (3.1) leads to certain predictions about the asymptotic behavior of fermion correlation functions in the so-called deformed (or anisotropic) $S U(2)$ Thirring model. The latter is described by the action (we use again euclidean notations)

$$
\mathcal{A}_{D T M}=\int d^{2} x\left\{\sum_{\sigma=\uparrow, \downarrow} \bar{\Psi}^{\sigma} \gamma_{\mu} \partial_{\mu} \Psi_{\sigma}+\frac{g_{0}}{2} J_{\mu} J_{\mu}+\frac{g_{\|}}{2} J_{\mu}^{3} J_{\mu}^{3}+2 g_{\perp} J_{\mu}^{+} J_{\mu}^{-}\right\}
$$

where $\Psi_{\uparrow}, \Psi_{\downarrow}$ is a doublet of Dirac Fermi fields, and

$$
J_{\mu}=\bar{\Psi} \gamma_{\mu} \Psi, \quad J_{\mu}^{A}=\bar{\Psi} \gamma_{\mu} \tau^{A} \Psi
$$

are their vector currents. The Pauli matrices $\tau^{A}=\left(\tau^{3}, \tau^{+}, \tau^{-}\right)$in (5.2) act on the "flavor" indices $\sigma=\uparrow, \downarrow$. The model $(\underline{5.1})$ is renormalizable, and its coupling constants $g_{\|}, g_{\perp}$ should be understood as "running" ones (the singlet coupling $g_{0}$ does not renormalize). The corresponding RG flow pattern is known as Kosterlitz-Thouless flow (see e.g. [21]). In particular, in the domain $g_{\|} \geq\left|g_{\perp}\right|$ all RG trajectories originate from the line $g_{\perp}=0$ of UV stable fixed points, and (5.1) indeed defines a quantum field theory. In this domain 
(which is the only one that we discuss here) each RG trajectory is uniquely characterized by the limiting value

$$
\bar{g}_{\|}=\lim _{L \rightarrow-\infty} g_{\|}(L)
$$

of the running coupling $g_{\|}(L)$ at extremely short distances ( $L$ stands for the logarithm of the length scale), i.e. the theory (5.1) depends on two dimensionless parameters $\bar{g}_{\|}$and $g_{0}$, besides the mass scale appearing through dimensional transmutation. The model (5.1) attracts much attention in condensed matter physics, e.g. because of its relation to the scaling limit of the Hubbard model (see e.g. [22]).

As is well known (see e.g. [23]), the model (5.1) can be bosonized in terms of the sine-Gordon field $\varphi(x)$, with $\beta$ in (2.1) related to $\bar{g}_{\|}$by

$$
\frac{1}{\beta^{2}}=1+\frac{2 \bar{g}_{\|}}{\pi}
$$

and a free massless boson $\omega(x)$. For the latter we assume conventional CFT normalization

$$
\langle\omega(x) \omega(0)\rangle=-2 \log (\mathrm{z} \overline{\mathrm{Z}})
$$

The chiral components of the Fermi fields

$$
\Psi_{\sigma}(x)=\frac{1}{\sqrt{2 \pi}}\left(\begin{array}{c}
\psi_{\sigma R}(x) \\
\psi_{\sigma L}(x)
\end{array}\right)
$$

are expressed in terms of these bosons as

$$
\begin{array}{llll}
\psi_{\downarrow L} & =\eta_{\downarrow} \mathcal{O}_{-\beta / 4}^{-1} \tilde{\mathcal{O}}_{\gamma / 4}^{1}, & \psi_{\downarrow R}=\eta_{\downarrow} \mathcal{O}_{\beta / 4}^{-1} \tilde{\mathcal{O}}_{-\gamma / 4}^{1}, \\
\psi_{\uparrow L}=\eta_{\uparrow} & \mathcal{O}_{\beta / 4}^{1} \tilde{\mathcal{O}}_{\gamma / 4}^{1}, & \psi_{\uparrow R}=\eta_{\uparrow} \mathcal{O}_{-\beta / 4}^{1} \tilde{\mathcal{O}}_{-\gamma / 4}^{1},
\end{array}
$$

where $\eta_{\sigma}=\eta_{\sigma}^{\dagger}$ are Klein factors $\left(\eta_{\uparrow}^{2}=\eta_{\downarrow}^{2}=1, \eta_{\uparrow} \eta_{\downarrow}=-\eta_{\downarrow} \eta_{\uparrow}\right)$. In (5.4) $\mathcal{O}_{a}^{ \pm 1}$ are nonlocal fields (2.4) of the sine-Gordon part of the bosonic theory, and $\tilde{\mathcal{O}}_{a}^{ \pm 1}$ are similar expressions in terms of the free field $\omega$, with $\beta$ replaced by $\gamma$, where

$$
\frac{1}{\gamma^{2}}=1+\frac{2 g_{0}}{\pi}
$$

These fields can also be written as

$$
\tilde{\mathcal{O}}_{a}^{n}(x)=\exp \left\{i\left(a-\frac{n}{4 \gamma}\right) \omega_{R}(\mathrm{z})-i\left(a+\frac{n}{4 \gamma}\right) \omega_{L}(\overline{\mathrm{z}})\right\}
$$


through the right and left moving chiral parts of $\omega(x)$. Note that each of the factors $\mathcal{O}$ and $\tilde{\mathcal{O}}$ in $(5.4)$ is nonlocal (they each have spin $\frac{1}{4}$ ), while the products appearing there are local Fermi fields.

As a result of the factorization (5.4) (known as spin-charge separation in condensed matter applications) the correlation functions of the fermions also factorize, for instance

$$
\left\langle\psi_{\sigma^{\prime} R}(x) \psi_{\sigma R}^{\dagger}(0)\right\rangle=\frac{i \delta_{\sigma^{\prime} \sigma}}{z} r^{\frac{1}{2}-\left(\gamma-\gamma^{-1}\right)^{2} / 4} \mathcal{G}_{-\beta / 4, \beta / 4}^{(1)}(r)
$$

where again $\mathrm{z}=\mathrm{x}+i \mathrm{y}, r=\sqrt{\mathrm{z} \overline{\mathrm{z}}}$. In writing (5.6) we have used (2.6), along with the well known form of the two-point correlation function of the free-field exponentials (5.5),

$$
\left\langle\tilde{\mathcal{O}}_{a^{\prime}}^{-n}(x) \tilde{\mathcal{O}}_{-a}^{n}(0)\right\rangle=\left(e^{i \pi \frac{\bar{z}}{\mathrm{z}}}\right)^{\frac{a n}{\gamma}} r^{-4 a^{2}-\frac{n^{2}}{4 \gamma^{2}}} \delta_{a^{\prime} a} .
$$

The short distance asymptotic form of this correlation function follows from (2.8),

$$
\lim _{r \rightarrow 0} \frac{\mathrm{Z}}{r} r^{2 d_{\psi}}\left\langle\psi_{\sigma^{\prime} R}(x) \psi_{\sigma R}^{\dagger}(0)\right\rangle=i \delta_{\sigma^{\prime} \sigma}
$$

with

$$
d_{\psi}=\frac{1}{2}+\frac{\left(\gamma-\gamma^{-1}\right)^{2}}{8}+\frac{\left(\beta-\beta^{-1}\right)^{2}}{8}
$$

This of course is nothing but our convention concerning the normalization of $\Psi_{\sigma}$ which we implicitly assumed in writing (5.4). On the other hand, using (2.16) with $n=1$ one can obtain its large-distance asymptotic,

$$
\left\langle\psi_{\sigma^{\prime} R}(x) \psi_{\sigma R}^{\dagger}(0)\right\rangle=\frac{i \delta_{\sigma^{\prime} \sigma}}{\mathrm{Z}} \frac{\mathbf{Z}_{1}(\beta / 4)}{\sqrt{2 \pi M}} r^{-\left(\gamma-\gamma^{-1}\right)^{2} / 4} e^{-M r}+O\left(e^{-3 M r}\right)
$$

where $M$ is the sine-Gordon soliton mass. While the functional form of (5.7) is well known [24], the coefficient $\mathbf{Z}_{1}(\beta / 4)$ constitutes a nontrivial prediction which follows from (3.1). Let us mention here that according to (5.7), in the case $\beta=\gamma=1$ (which corresponds to the symmetric model (5.1) with $g_{0}=0$ and $g_{\|}=g_{\perp}>0$ ) one has

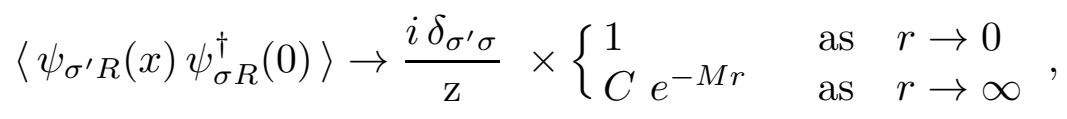

with

$$
C=\frac{\Gamma(1 / 4)}{2^{\frac{5}{4}} \sqrt{\pi}} \exp \left\{\int_{0}^{\infty} \frac{d t}{t} \frac{\sinh ^{2}(t / 2) e^{-t}}{\sinh (2 t) \cosh (t)}\right\}=0.921862 \ldots .
$$

The numerical coefficients in the short- and long-distance asymptotics in (5.8) are remarkably close; this suggests that in this symmetric case the leading term in the long-distance 
asymptotic (5.8) may be rather good approximation for the correlation function at all scales.

Eq. (5.8) can be translated to asymptotic formulae for the fermion correlation functions in the half-filled Hubbard chain

$$
\mathbf{H}_{H u b}=-t \sum_{j=-\infty}^{+\infty} \sum_{\sigma=\uparrow, \downarrow}\left(c_{j, \sigma}^{\dagger} c_{j+1, \sigma}+c_{j+1, \sigma}^{\dagger} c_{j, \sigma}\right)+U \sum_{j=-\infty}^{+\infty}\left(n_{j, \uparrow}-\frac{1}{2}\right)\left(n_{j, \downarrow}-\frac{1}{2}\right) .
$$

In (5.10) $c_{\sigma, j}, c_{\sigma, j}^{\dagger}$ are the Fermi operators,

$$
\left\{c_{j, \sigma}^{\dagger}, c_{j^{\prime}, \sigma^{\prime}}\right\}=\delta_{\sigma \sigma^{\prime}} \delta_{j j^{\prime}}
$$

and $n_{j, \sigma}=c_{j, \sigma}^{\dagger} c_{j, \sigma}$. As is known [25,26,27,28, 29, in the symmetric case $\left(g_{0}=0\right.$ and $g_{\|}=g_{\perp}>0$ ), the field theory (5.1) describes the scaling limit of this chain. If one sends $U \rightarrow+0$, the correlation length

$$
R_{c}=\frac{\pi}{2} \sqrt{\frac{t}{U}} e^{\frac{2 \pi t}{U}}
$$

diverges, and the correlation functions of (5.10) at large lattice separations assume certain scaling forms. In particular, if $\left|j-j^{\prime}\right| \gg 1$ the equal-time fermion correlator can be written as

$$
\left\langle c_{j^{\prime}, \sigma^{\prime}} c_{j, \sigma}^{\dagger}\right\rangle \rightarrow \delta_{\sigma^{\prime} \sigma} \frac{\sin \left(\frac{\pi}{2}\left(j^{\prime}-j\right)\right)}{\pi\left(j^{\prime}-j\right)} F\left(\left|j^{\prime}-j\right| / R_{c}\right)
$$

where $F(0)=1$. The scaling function $F(\tau)$ here is related directly to the field-theoretic correlation function (5.6) with $\beta=\gamma=1$. Therefore Eq.(5.8) leads to the following prediction for the large-distance asymptotic of the scaling function $F$ in (5.11),

$$
F(\tau)=C e^{-\tau}+O\left(e^{-3 \tau}\right) \quad \text { as } \quad \tau \rightarrow \infty
$$

where $C$ is the constant (5.9).

\section{Discussion}

Conjecture (3.1) for the factor $\mathbf{Z}_{n}(a)$ in (2.12) is the main statement of this paper. In certain sense it extends the proposal of [4] about vacuum expectation values of $\mathcal{O}_{a}^{0}(x) \equiv$ $\exp (i a \varphi(x))$ to the case of fields $\mathcal{O}_{a}^{n}(x)$ carrying nonzero topological charge $n$. In this connection we would like to add the following remark. 
As was shown in [5], the vacuum expectation values

$$
\left\langle v a c\left|e^{i a \varphi}\right| v a c\right\rangle=\sqrt{\mathbf{Z}_{0}(a)}
$$

obey the interesting analytic relation

$$
\sqrt{\mathbf{Z}_{0}(a)}=R_{0}(a) \sqrt{\mathbf{Z}_{0}(-\mathcal{Q}-a)},
$$

where $\mathcal{Q}=\frac{1}{\beta}-\beta$, and the amplitude

$$
\begin{gathered}
R_{0}(a)=(\Lambda)^{-2 \mathcal{Q}(2 a+\mathcal{Q})} \frac{\Gamma\left(1+\frac{2 a}{\beta}+\frac{\mathcal{Q}}{\beta}\right) \Gamma(1-2 a \beta-\mathcal{Q} \beta)}{\Gamma\left(1-\frac{2 a}{\beta}-\frac{\mathcal{Q}}{\beta}\right) \Gamma(1+2 a \beta+\mathcal{Q} \beta)}, \\
\Lambda=\frac{\sqrt{\pi} M \Gamma\left(\frac{3}{2}+\frac{\xi}{2}\right)}{2 \Gamma\left(1+\frac{\xi}{2}\right)}\left(\frac{1+\xi}{\xi}\right)^{\xi}
\end{gathered}
$$

can be related to the "Liouville reflection amplitude" [30] by analytic continuation $\beta \rightarrow$ ib. Although our understanding of the "reflection relation" (6.1) is still far from being satisfactory, it has proved to be very useful in determining vacuum expectation values of other fields in the sine-Gordon [31] and in other integrable models [5,6.7]. It is possible to check that $\mathbf{Z}_{n}(a)$ in Eq.(3.1) satisfies a similar relation

$$
\sqrt{\mathbf{Z}_{n}(a)}=R_{n}(a) \sqrt{\mathbf{Z}_{n}(-\mathcal{Q}-a)}
$$

with

$$
R_{n}(a)=(\Lambda)^{-2 \mathcal{Q}(2 a+\mathcal{Q})} \frac{\Gamma\left(1+\frac{2 a}{\beta}+\frac{\mathcal{Q}}{\beta}+\frac{n}{2 \beta^{2}}\right) \Gamma(1-2 a \beta-\mathcal{Q} \beta+n / 2)}{\Gamma\left(1-\frac{2 a}{\beta}-\frac{\mathcal{Q}}{\beta}+\frac{n}{2 \beta^{2}}\right) \Gamma(1+2 a \beta+\mathcal{Q} \beta+n / 2)} .
$$

At the moment we are not in possession of any clear interpretation of the "reflection amplitude" (6.4), neither in terms of Liouville nor any other CFT, nor even as a natural analytic interpolation of the "normalization factors" of [7] (note that (6.4) does not have $n \rightarrow-n$ symmetry, which the Coulomb-gas integrals defining the corresponding normalization factors clearly do, thus indicating a significant ambiguity in such analytic interpolation for $n \neq 0$ ). Nonetheless, Eq. (6.3) could be helpful in the search for generalizations of (3.1).

\section{Acknowledgments}

The authors acknowledge helpful discussions with Alexei Tsvelik. They are also indebted to Chris Hooley for careful reading of the manuscript. S.L. heartily thanks the organizers of the research program "New Theoretical Approaches to Strongly Correlated Systems", at the Isaac Newton Institute for Mathematical Sciences, Cambridge, where parts of this work were done, for their kind hospitality. He has especially benefited from discussions with Fabian Essler.

This research is supported in part by DOE grant \#DE-FG02-96ER10919. 


\section{References}

[1] Smirnov, F.A.: Form-factors in Completely Integrable Models of Quantum Field Theory. Singapore: World Scientific (1992)

[2] Vergeles, S. and Gryanik., V.: Two-dimensional Quantum Field Theories having exact solutions. Yadern. Fiz. 23, pp. 1324-1334 (1976) (in Russian);

Karowski, M., Weisz, P.: Exact form factors in (1+1)-dimensional field theoretic models with solution behavior. Nucl. Phys. B139, pp. 455-476 (1978);

Zamolodchickov, A.B.: Quantum sine-Gordon model. The soliton form-factors. ITEP45-1977, Preprint ITEP, 12pp. (1977)

[3] Zamolodchikov, Al.B.: Two-point correlation function in scaling Lee-Yang model. Nucl. Phys. B348, pp. 619-641 (1991);

Acerbi C., Mussardo G., and Valleriani, A.: Form-factors and correlation functions of the stress - energy tensor in massive deformation of the minimal models $E_{(N)}^{(1)} \times E_{(N)}^{(1)} / E_{(N)}^{(2)}$. Int. J. Mod. Phys. A11, pp. 5327-5364 (1996);

Balog, J. and Niedermaier, M.: Off-shell dynamics of the O(3) NLS model beyond monte carlo and perturbation theory. Nucl. Phys. B500, pp. 421-461 (1997)

[4] Lukyanov, S. and Zamolodchikov, A.: Exact expectation values of local fields in the quantum sine-Gordon model. Nucl. Phys. B493, pp. 571-587 (1997)

[5] Fateev, V., Lukyanov, S., Zamolodchikov, A. and Zamolodchikov, Al.: Expectation values of boundary fields in the boundary sine-Gordon model. Phys. Lett. B406, pp. 83-88 (1997);

Expectation values of local fields in the Bullough-Dodd model and integrable perturbed Conformal Field Theories. Nucl. Phys. B516, pp. 652-674 (1998)

[6] Baseilhac, P. and Fateev, V.A.: Expectation values of local fields for a two-parameter family of integrable models and related perturbed Conformal Field Theories. Nucl. Phys. B532, pp. 567-587 (1998)

[7] Fateev, V.A.: Normalization factors in Conformal Field Theory and their applications. Mod. Phys. Lett. A15, pp. 259-270 (2000)

[8] Lukyanov, S.: Free field representation for massive integrable models. Commun. Math. Phys. 167, No.1, pp. 183-226 (1995) ;

Form-factors of exponential fields in the sine-Gordon model. Mod. Phys. Lett. A12, pp. 2543-2550 (1997)

[9] Babujian, H., Fring, A., Karowski, M. and Zapletal, A.: Exact form-factors in integrable quantum field theories: the sine-Gordon model. Nucl. Phys. B538, pp. 535-586 (1999);

Delfino, G.: Off critical correlations in the Ashkin-Teller model. Phys. Lett. B450, pp. 196-201 (1999) 
[10] Fateev, V.A. and Zamolodchikov, A.B.: Parafermionic currents in the two-dimensional Conformal Quantum Field Theory and self-dual critical points in $Z_{N}$ invariant statistical systems. Zh. Eksp. Teor. Fiz. 89, pp. 380-399 (1985)

[11] Lukyanov, S. and Zamolodchikov, A.: To appear

[12] Coleman, S.: The quantum sine-Gordon equation as the Massive Thirring model. Phys. Rev. D11, pp. 2088-2097 (1975)

[13] Mandelstam, S.: Soliton operators for the quantized sine-Gordon equation. Phys.Rev. D11, pp. 3026-3030 (1975)

[14] Bernard, D. and LeClair, A.: Residual quantum symmetries of the restricted SineGordon theories. Nucl. Phys. B340, pp. 721-751 (1990)

[15] Baxter, R.J.: Exactly Solved Models in Statistical Mechanics. London: Academic Press (1982)

[16] Luther, A.: Eigenvalue spectrum of interacting massive fermions in one dimension. Phys. Rev. B14, 5, 2153-2159 (1976)

[17] Johnson, J.D., Krinsky, S. and McCoy, B.M.: Vertical-arrow correlation length in the eight-vertex model and the low-lying excitation of the X-Y-Z Hamiltonian. Phys. Rev. A8, 2526-2547 (1973)

[18] Lukyanov, S.: Low energy effective Hamiltonian for the XXZ spin chain. Nucl. Phys. B522, pp.533-549 (1998)

[19] Foda, O., Iohara, K., Jimbo, M., Kedem R., Miwa, T. and Yan, H.: An elliptic quantum algebra for SL(2). Lett. Math. Phys. 32, pp. 259-268 (1994)

[20] Lukyanov, S. and Pugai, Ya.: Bosonization of ZF algebras: Direction toward deformed Virasoro algebra. J. Exp. Theor. Phys. 82, pp. 1021-1045 (1996)

[21] Itzykson, C. and Drouffe, J.-M.: Statistical Field Theory. Vol.1: Cambridge University Press (1989)

[22] Controzzi, D., Essler, F.H.L. and Tsvelik, A.M.: Dynamical properties of onedimensional Mott insulators. To appear in the proceedings of Newton Institute EuroConference on Strongly Correlated Electron Systems: Novel Physics and New Materials, Cambridge, England, 26-30 June 2000 (cond-mat/0011439)

[23] Gogolin, A.O., Nersesyan, A.A. and Tsvelik, A.M.: Bosonization and Strongly Correlated Systems. Cambridge University Press (1999)

[24] Essler, F.H.L. and Tsvelik, A.M.: Private communication

[25] Filev, V.M.: Spectrum of two-dimensional relativistic model. Teor. i Mat. Fiz. 33, pp. 119-124 (1977)

[26] Emery, V.J., Luther, A. and Peschel, I.: Solution of the one-dimensional electron gas on a lattice. Phys. Rev. B13, pp.1272-1276 (1976)

[27] Melzer, E.: On the scaling limit of the 1-d Hubbard model at half filling. Nucl. Phys. B443 [FS], pp. 553-564 (1995) 
[28] Woynarovich, F. and Forgacs, P.: Scaling limit of the one-dimensional attractive Hubbard model: The half-filled band case. Nucl. Phys. B498, pp.65-603 (1997)

[29] Korepin, V.E. and Essler, F.H.L.: $S U(2) \otimes S U(2)$ invariant scattering matrix of the Hubbard model. Nucl. Phys. B426, pp. 505-533 (1994)

[30] Zamolodchikov, A.B. and Zamolodchikov, Al.B.: Structure Constants and Conformal Bootstrap in Liouville Field Theory. Nucl. Phys. B477, 577-605 (1996)

[31] Fateev, V., Fradkin, D., Lukyanov, S., Zamolodchikov, A. and Zamolodchikov, Al.: Expectation values of descendent fields in the sine-Gordon model. Nucl. Phys. B540, pp. 587-609 (1999) 